\title{
Perspective
}

PERSPECTIVE Actualité en histoire de l'art

Comptes rendus | 2009

\section{Alexandre G. Mitchell, Greek Vase-Painting and the Origins of Visual Humour, New York, Cambridge University Press, 2009 et David Walsh, Distorted Ideals in Greek Vase-Painting: The World of Mythological Burlesque, New York, Cambridge University Press, 2009}

\section{Maria Pipili}

\section{OpenEdition}

\section{Journals}

Édition électronique

URL : http://journals.openedition.org/perspective/2765

DOI : $10.4000 /$ perspective. 2765

ISSN : 2269-7721

Éditeur

Institut national d'histoire de l'art

Référence électronique

Maria Pipili, « Alexandre G. Mitchell, Greek Vase-Painting and the Origins of Visual Humour, New York, Cambridge University Press, 2009 et David Walsh, Distorted Ideals in Greek Vase-Painting: The World of Mythological Burlesque, New York, Cambridge University Press, $2009 »$, Perspective [En ligne], Comptes rendus, mis en ligne le 02 août 2013, consulté le 01 octobre 2020. URL : http://

journals.openedition.org/perspective/2765; DOI : https://doi.org/10.4000/perspective.2765

Ce document a été généré automatiquement le 1 octobre 2020. 


\section{Alexandre G. Mitchell, Greek Vase- Painting and the Origins of Visual Humour, New York, Cambridge University Press, 2009 et David Walsh, Distorted Ideals in Greek Vase- Painting: The World of Mythological Burlesque, New York, Cambridge University Press, 2009}

Maria Pipili

\section{RÉFÉRENCE}

Alexandre G. Mitchell, Greek Vase-Painting and the Origins of Visual Humour, New York, Cambridge University Press, 2009.

David Walsh, Distorted Ideals in Greek Vase-Painting: The World of Mythological Burlesque, New York, Cambridge University Press, 2009. 
1 Les deux livres, publiés simultanément par Cambridge University Press et reprenant plus ou moins des thèses de doctorat, sont consacrés à la figure humoristique dans la Grèce ancienne, sujet attrayant sur lequel on ne disposait jusqu'à présent d'aucune étude systématique. Le thème est abordé à travers les vases peints (presque
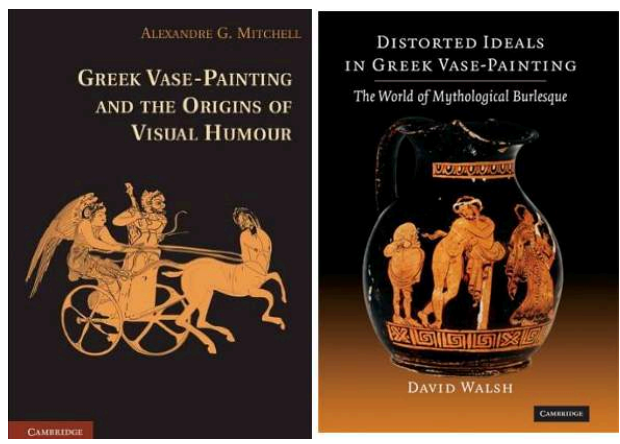
exclusivement athéniens et béotiens dans l'ouvrage de Mitchell), principaux supports d'images aux époques archaïque et classique. S'attachant à mettre au jour la fonction sociale de l'humour, Alexandre Mitchell se penche sur des scènes humoristiques inspirées aussi bien de la vie quotidienne que des mythes, qu'il relie à la liberté d'expression introduite par la démocratie athénienne. David Walsh s'intéresse quant à lui au burlesque et à l'irrévérence dans le domaine religieux, tels que l'expriment le théâtre comique et les vases d'Italie du Sud, offrant en appendice un très utile catalogue des œuvres étudiées. Les deux ouvrages se recoupent inévitablement sur certains sujets, comme la satire et la parodie comique ou encore les caricatures du sanctuaire du Cabirion de Béotie. Chacun suit en revanche son propre objectif et répond à une perspective spécifique. Ainsi peuvent-ils se lire de façon complémentaire par tous ceux qu'intéressent à la culture et à la société de la Grèce antique, où la figure humoristique tenait une place non négligeable. 